\title{
Detecting Distributed Network Traffic Anomaly with Network-Wide Correlation Analysis
}

\author{
Li Zonglin, Hu Guangmin, Yao Xingmiao, and Yang Dan \\ Key Lab of Broadband Optical Fiber Transmission and Communication Networks, \\ University of Electronic Science and Technology of China (UESTC), Chengdu 610054, China \\ Correspondence should be addressed to Li Zonglin, lizonglin@uestc.edu.cn
}

Received 22 October 2007; Accepted 20 August 2008

Recommended by Rocky Chang

Distributed network traffic anomaly refers to a traffic abnormal behavior involving many links of a network and caused by the same source (e.g., DDoS attack, worm propagation). The anomaly transiting in a single link might be unnoticeable and hard to detect, while the anomalous aggregation from many links can be prevailing, and does more harm to the networks. Aiming at the similar features of distributed traffic anomaly on many links, this paper proposes a network-wide detection method by performing anomalous correlation analysis of traffic signals' instantaneous parameters. In our method, traffic signals' instantaneous parameters are firstly computed, and their network-wide anomalous space is then extracted via traffic prediction. Finally, an anomaly is detected by a global correlation coefficient of anomalous space. Our evaluation using Abilene traffic traces demonstrates the excellent performance of this approach for distributed traffic anomaly detection.

Copyright () 2009 Li Zonglin et al. This is an open access article distributed under the Creative Commons Attribution License, which permits unrestricted use, distribution, and reproduction in any medium, provided the original work is properly cited.

\section{Introduction}

Network traffic anomaly is referred to as a situation such that traffic deviates from its normal behavior, while distributed network traffic anomaly is a traffic abnormal behavior involving multiple links of a network and caused by the same source. There are many reasons that can cause distributed network traffic anomaly, such as DDoS attack, flash crowd, sudden shifts in traffic, worm propagation, network failure, network outages, and so forth. Any of these anomalies will seriously impact the performance of network.

Usually, there are not any obvious features of anomalies in individual links for distributed network traffic anomaly, that is, compared with background traffic of backbone network, even its normal changes, anomalous traffic may be unnoticeable so that detection based on information collected from single link is very difficult. However, the sum of anomalous traffic on many links can be prevailing. If we put multitraffic singles together and apply networkwide anomaly detection to them, the relationship between traffic would help to reveal anomaly. Principle component analysis (PCA) is an existing statistical-analysis technique;
Lakhina et al. [1,2] applied it as a network-wide detection method to the field of traffic anomaly detection. It follows that decomposing overall traffic into two disjoint parts based on correlation across links or origin-destination (OD) flows, respectively, corresponds to normal space and anomalous space. Traffic with less correlation is considered as anomalous space, the energy of anomalous space; is then compared with a threshold to diagnosis anomaly.

The distributed traffic anomalies caused by the same source usually have some similar features in time or frequency domain. These similarities contribute to strong correlation between anomalous flows. Since PCA-based methods deal with the anomalous space that lacks correlation, they are prone to suffer from false negative. Although the volume of individual anomaly is small, anomalous flows in many links exhibit inherent correlations. This fact should be useful for detection. Drawing on the change of correlation between network-wide anomalous space lends itself to bypass the limitation of PCA-based methods. In this paper, we propose a method to detect distributed traffic anomaly with network-wide correlation analysis of instantaneous parameters. First traffic signals' instantaneous 
parameters are computed; and their network-wide anomalous space is then extracted via traffic prediction; finally, global correlation coefficient as a measure of the correlation between anomalous space is calculated to reveal anomaly.

The contributions of this paper are as follows.

(i) We perform detection on instantaneous amplitude and instantaneous frequency of traffic signal, which can reveal anomalies by its characteristics of time and frequency domain. To improve computation speed of instantaneous parameters, we propose a fast algorithm of instantaneous parameters computation for anomaly detection.

(ii) We divide anomalous space by means of comparing the actual instantaneous parameters of OD flows with the predictions to overcome limitation of PCA in failing to detect the anomalies with strong correlations.

(iii) Targeting at the characteristics of distributed traffic anomaly, we deploy detection by correlation analysis of amplitude and frequency between anomalous space, rather than volume, which can detect small anomaly in single link.

\section{Related Work}

Network traffic anomaly detection method can be classified into single node and multinodes detection by traffic number being analyzed. Based on whether to take into account the relationship between traffic, multinodes detection can be further differentiated between distributed detection and network-wide detection.

Distributed detection [3-10] is to select some nodes in the network to construct subdetection networks. First, each node deploys simple and fast local detection by selfcollected information; second, exchange detecting results of each node through a certain communication mechanism; then, synthesize the results of partial or all nodes to determine whether anomaly occurs. Some related systems or architecture have been reported, for instance, distributed attack detection system (DAD) $[4,5]$, Cooperative Intrusion Traceback and Response Architecture (CITRA) [6, 7], and so on. In addition, some try to deploy local detection by frequency domain analysis, as shown in [11]. This collaborative distributed detection, that determines anomaly by detection results on many nodes, overcomes the limit of detecting only by one single node and increases detection accuracy effectively. However, its final detection result still depends on local result of each node to a great extent, whereas distributed network anomaly does not present obvious feature on single node, which makes it hard to detect one of them.

Being different from the former distributed detection which tends to detect at different position independently, network-wide detection is a method that analyzes all traffic signals together and exposes anomaly through relationship between traffic. Diagnosis anomaly in network-wide perspective was firstly reported in the works of Lakhina et al. $[1,2]$; they perform PCA to analyze the relationship between volume of all links or OD flows, in order to divide anomalous part from traffic. In 2005, Lakhina et al. [12] proposed an anomaly detection method by applying PCA to the feature distribution of network-wide traffic, and a DDoS attack detection method using multiway PCA [13]. Li et al. [14] introduced a method combining traffic sketch and subspace for network-wide anomaly detection. Yuan and Mills [15] defined a weight vector and discovered congestion on many links by cross-correlation analysis. Huang et al. [16] detected network disruption via performing PCA to network-wide routing updates data.

Most of existing network-wide detection methods are based on PCA. The main advantage of these methods is the use of the relationship among overall traffic, and can detect some anomalies effectively, especially abrupt change of traffic at local point. The basic idea of PCA is to treat traffic which are highly correlated as normal space and only analyze the remaining anomaly space. However, distributed traffic anomalies caused by the same source possess high correlation with each other, and they are prone to be divided into normal space by PCA. Therefore, PCA-based method may suffer from false negative in detecting distributed network traffic anomaly. Furthermore, these methods still determine anomaly only by the value of traffic volume, which leads to the difficulties in detecting relatively small distributed anomalous traffic from normal ones. In this paper, we divide anomalous space by comparing predictions of traffic instantaneous parameters with real value, and make use of the variation degree of correlation between anomalous space, rather than volume, to infer anomaly.

Signal process technique has been widely used in traffic anomaly detection for single node. Cheng et al. [17] found that the PSD of normal TCP flows exhibit periodicity while the PSD of DoS attack flow is not. Hussain et al. [18] utilized the difference of PSD in lower frequency band to classify the attacks as single or multisource. Chen and Hwang [11] compared the PSD of normal traffic with attack in lower frequency band with the aim of periodic pulsing DDoS attack detection. The PSD of signal illustrates the proportion of every frequency component as a whole, however it lacks local information, and cannot be more specific about the time each frequency component is involved in, while it is more important to nonstationary traffic signals whose frequency components are time varying. The instantaneous parameters can provide information about amplitude and frequency of nonstationary signal in every time point and how they change with time. Wang et al. [19] used Hilbert-Huang transform [20] to acquire the instantaneous frequency of traffic as an outline of normal behavior for single link. In this paper, we use both instantaneous parameters of OD flow, namely, instantaneous frequency and instantaneous amplitude, and divide anomalous space for each of them.

The main difference between our method and [19] is that, first, the method proposed by [19] is used for single node detection, it attempted to find anomaly based on obvious change of traffic instantaneous frequency, however the variation of instantaneous frequency caused by distributed anomaly traffic on individual link is potentially small, the detection method would be hampered by this fact. Whereas, we analyze network-wide OD flows, and use the change of 
correlation caused by the effect of alteration simultaneously across multiple traffic data, to circumvent the difficulty caused by individual anomaly with small variation in instantaneous frequency. Second, the analysis in [19] was only for traffic instantaneous frequency. Since anomalous traffic may cause different impact on instantaneous frequency and instantaneous amplitude of background traffic, there might exist false negative in detection from instantaneous frequency or amplitude solely. Instead, we use instantaneous amplitude as well as instantaneous frequency so as to achieve a better detection performance.

\section{Distributed Network Traffic Anomalies Detection}

Distributed network traffic anomalies caused by the same source usually have some similar features. For instance, the anomalies arose by same attack event, commonly generated by specific tools, might possess some similarities in their start time, lasting time, interval time, type and frequency characteristic, and so forth; likewise, the alternative distributed traffic anomalies caused by nonattack reasons, like outages, might result in the flows that traverse the location of anomalous event change simultaneously. These similarities both in time and frequency domain contribute to the strong correlation between anomalous flows.

The previous anomaly detection methods usually make use of the difference between individual anomaly and the normal pattern to derive judgment. However, they generally fail to detect the anomalies on individual links which are relatively small. The alteration of single anomalous flow is unnoted, while the variational tendency of multiple anomalous flows in time or frequency domain is easy to be captured, and by means of this collectively variational tendency, can conquer the difficulties resulting from small single anomaly. Therefore, the concept of correlation can be used to characterize the relationship between multiflows when they change simultaneously.

As the correlation of anomalous flows is not only exhibited in time domain, but also reflected in frequency domain, it is advantageous to consider more kinds of features of anomalous flows both in time and frequency domains for correlation analysis to reveal anomaly. Instantaneous parameters (i.e., both instantaneous amplitude and instantaneous frequency) are physical parameters, which capture transient characteristic of signal, and characterize it in different ways. In this sense, we perform correlation analysis on the two instantaneous parameters of anomalous flows to identify anomalies more extensively.

Besides the correlation of distributed anomalous flows, there still exists correlation between normal traffic, such as the similar diurnal and weekly pattern. Accordingly before we perform correlation analysis on anomalies, it is necessary to eliminate the influence of correlation between normal traffics to avoid the impact on detection result, it is equivalent to extract anomalous space from the whole traffic signal.

The detection steps are depicted in Figure 1. Firstly, we compute instantaneous parameters of every OD flows to get

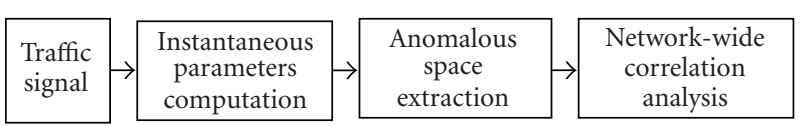

FIGURE 1: Distributed network traffic anomaly detection steps.

their instantaneous amplitude and frequency; then model the instantaneous parameters with corresponding time series models, the difference between actual data and predictions is used to approximate the anomalous space which includes abnormal flows; finally, network-wide correlation analysis is performed on the anomalous space and detect distributed traffic anomaly by the variation degree of correlation. The computation of instantaneous parameters, extraction of anomalous space, and network-wide correlation analysis will be elaborated, respectively, in Sections 4, 5, 6 .

\section{Instantaneous Parameters and Fast Algorithm}

4.1. Instantaneous Parameters. Traffic signal is nonstationary, it varies with time, so does its frequency content. The instant characteristic of nonstationary signal is generally captured by instantaneous parameters (including instantaneous amplitude (IA), instantaneous frequency (IF)), which decompose the information of amplitude and frequency, and do not change the nature of signal, but rather to set up reflections of different aspects. Instantaneous parameters tend to reveal some characteristics of signal that are covered by usual time description. The definitions of instantaneous parameters are as follow: for any continue time signal $X(t)$, we can get its Hilbert transformation: $Y(t)=(1 / \pi) \int_{-\infty}^{+\infty} X(\tau) /(t-\tau) d \tau$, then resolve signal $Z(t)$ is obtained by $Z(t)=X(t)+i Y(t)=$ $a(t) e^{j \theta(t)}$, where $\theta(t)=\arctan (Y(t) / X(t))$ is the phases function of $Z(t)$. The instantaneous amplitude of $Z(t)$ is computed by:

$$
a(t)=\left[X(t)^{2}+Y(t)^{2}\right]^{1 / 2} .
$$

Instantaneous frequency $\omega(t)$ is denoted as

$$
\omega(t)=\frac{d \theta(t)}{d t} .
$$

\subsection{Fast Algorithm for Instantaneous Parameters Computa- tion. Anomaly detection is usually required to be processed online. In computation of instantaneous parameters, a whole traffic series is needed for convolution, however it cannot meet the need of real-time operation. Accordingly, a sliding window can be used in practical calculation, to move along the traffic and intercept data from it. While the window is sliding, the two data sets, intercepted, respectively, before and after window moves, always have a same part, and there would be a lot of redundant results if we compute the same part twice. So it is convenient to store this part of instantaneous parameters in advance, and only compute the new data intercepted by the window to avoid repeating calculation and improve the detection speed.}




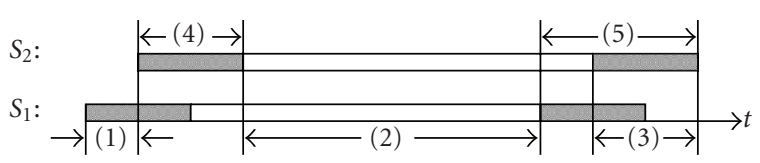

FIGURE 2: Fast algorithm of instantaneous parameters computation.

Let $S_{1}$ be the traffic data set intercepted by sliding window at certain time, and the length of window is $N$, the kernel of the Hilbert transform $1 /(\pi t)$, which can be considered as a filter with the length of $2 L$, then the Hilbert transform of $S_{1}(k)$ can be written as

$$
H S_{1}(k)=\sum_{i=-L}^{L} \frac{S_{1}(k)}{(k-i) \pi}, \quad k=0,1, \ldots, N .
$$

When $k-i<0$, namely $0 \leq k \leq L$, the data of $S_{k}$ in this section are out of range and demand process separately, this section is at the beginning of the signal. When $k-i>N$, namely $k-i>N$, the data in this section are out of range and demand process separately, this section is at the end of the signal. When $L \leq k \leq N-L$, the data in this section do the normal convolution.

As moving along the traffic data, the sliding window samples the data to get another signal $S_{2}$ every time lapse of $\Delta T$, as depicted in Figure 2, it is composed as follows:

$$
S_{2}(K)= \begin{cases}S_{1}(k-\Delta N), & 0 \leq k \leq(N-\Delta N), \\ \text { new input data, } & (N-\Delta N)<k \leq N .\end{cases}
$$

The data of $S_{1}$ in the section $L+\Delta N \leq k \leq N-L$ are the same as the data of $S_{2}$ in the section $L \leq k \leq N-L-\Delta N$, so the instantaneous parameters $\operatorname{IP}_{1}(k)$ and $\mathrm{IP}_{2}(k)$ of this part are the same, as represented in Figure 2(2). The number of the same points is $M=N-2 L-\Delta N$. Therefore, as long as $N>L$, we only need to compute the instantaneous parameters of $S_{2}$ in the section of $k \in[0, L] \cup[N-(L+$ $\Delta N), N]$. 4 steps.

The fast calculation of instantaneous parameters includes

(i) Compute the instantaneous parameters $\operatorname{IP}_{1}(k)$ of signal $S_{1}$, and store $k \in[L, N-L-\Delta N]$ part of $\operatorname{IP}_{1}(k)$ to be the section of $\operatorname{IP}_{2}(k)$ for $k \in[L, N-L-\Delta N]$, which is represented in Figure 2(2).

(ii) According to the principle of data periodic repetition which deals with data beyond the boundary, we pick up the part of $k \in[N-L, N]$ from $S_{2}$, and convolute with filter to get the section of $\operatorname{IP}_{2}(k)$ for $k \in[0, L)$, as it shown in Figure 2(4).

(iii) Pick up the part of $k \in[0, L]$ from $S_{2}$, and convolute with filter to get the section of $\operatorname{IP}_{2}(k)$ for $k \in(N-$ $(L+\Delta N), N)$, as it shown in Figure 2(5).

(iv) Synthesizing three steps mentioned before, we can get the whole instantaneous parameters $\operatorname{IP}_{2}(k)$ of $S_{2}$.

The fast algorithm of instantaneous parameters based on sliding window technology adds an array with the length of

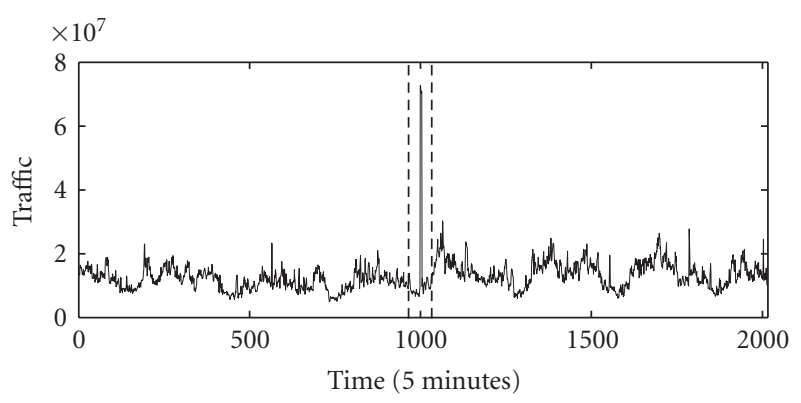

(a) Adding one anomaly in no.26 OD flow (between vertical dash lines)

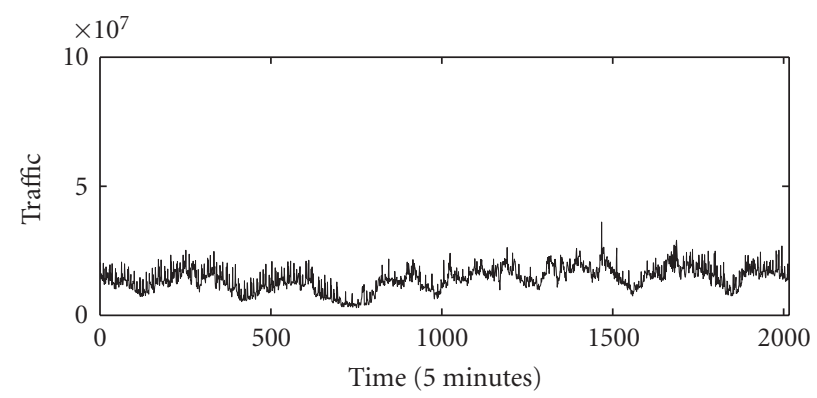

(b) No.50 OD flow unstained

Figure 3: Anomaly in a single flow.

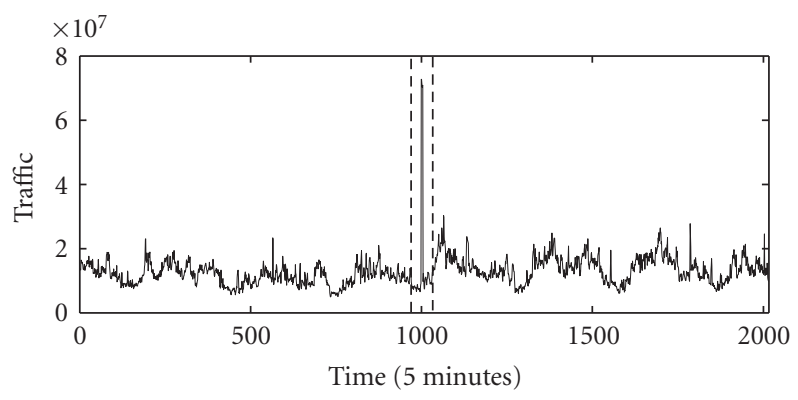

(a) Adding one anomaly in no.26 OD flow (between vertical dash lines)

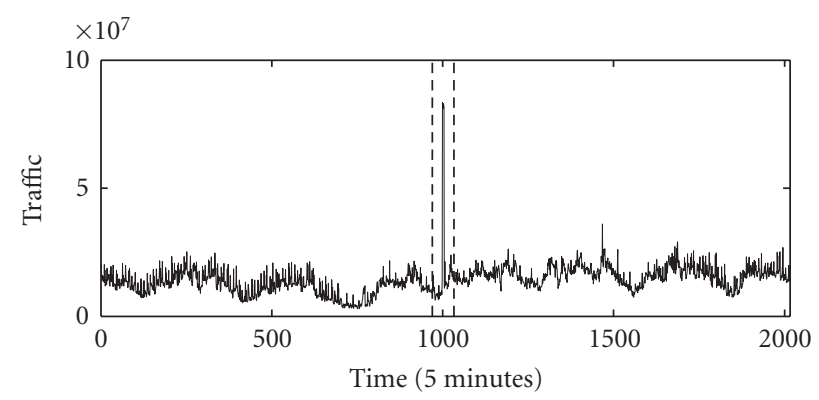

(b) Adding one anomaly in no.50 OD flow (between vertical dash lines)

Figure 4: Two anomalies in two flows.

$M(M=N-2 L-\Delta N)$, to record the same part between $\mathrm{IP}_{1}(k)$ and $\mathrm{IP}_{2}(k)$, by comparison with normal computation. When calculating $\operatorname{IP}_{2}(k)$, the same part with $\operatorname{IP}_{1}(k)$ can be transferred directly to the result to improve the computation speed of instantaneous parameters. 


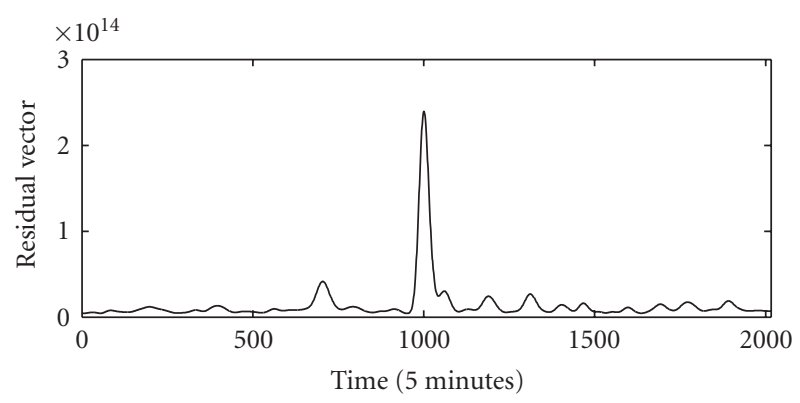

FIGURE 5: PCA for one anomalies in two flows.

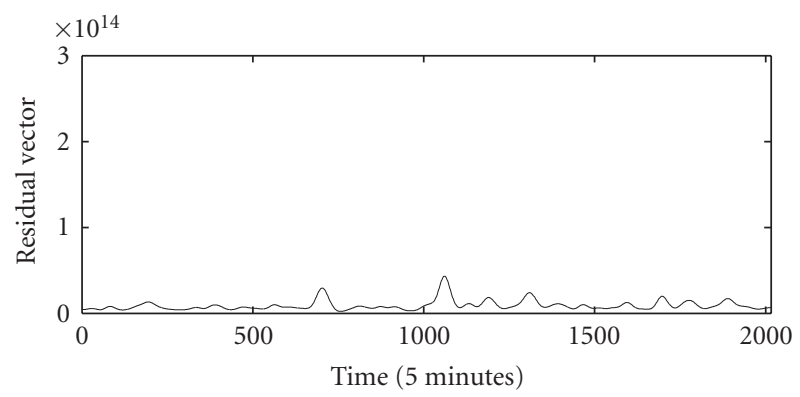

FIGURE 6: PCA for two anomalies in two flows.

\section{Anomalous Space Extraction}

The extraction of anomalous space from traffic signal is implemented via getting rid of normal traffic behavior. Most of network-wide anomaly traffic detection methods are PCAbased method, they draw on PCA to divide traffic into normal and abnormal space, the normal part is determined while they have strong temporal trend among links or OD flows. It performs well in detecting abrupt change in the local of single traffic, but may be limited to the case of distributed traffic anomaly, for the anomalies with strong correlation are possibly divided into normal space. We will illustrate it by changing the number of anomalous flows.

Figure 3 is the traffic of no. 26, 50 OD flows of Abilene network (more detail in Section 7.1) in the 3rd week. In Figure 3(a), we inject one anomaly to $26 \mathrm{OD}$ flow with five times of the mean of it, from 1000 to 1004 sample point, which corresponds to the spike and can be easily visually isolated. 50th OD flow is unstained. The anomalous space derived by PCA is depicted in Figure 5, and the abrupt change of 26th OD flow is correctly partitioned. In the same way, we inject another anomaly with 5 times of its mean and the same lasting time on 50th OD flow, as shown in Figure 4(b). There are similarities between two anomalies in the beginning, lasting time, and the change of volume. The outcome of PCA for the two anomalies is shown in Figure 6. It shows that the anomalies nearby the 1000th sample point are not divided into the anomalous space, instead they are considered as the normal due to the strong correlation. Therefore, PCA method cannot separate anomalous space for distributed traffic anomaly with strong correlation.
Observing from normal OD flows, traffic usually consists of normal part and the part representing some random factors, which might be the result of accidental behavior of users when there exists no anomaly. Owing to the similar daily and weekly pattern of traffic, the normal part must have some correlation, if the behavior of normal traffic is separated, the residual of different OD flows should not have correlation, which means that the residual traffic are independent of each other. While anomaly occurs, anomalous flows are of strong correlation. For this reason, the correlation of normal traffic is necessary to be restrained. ARIMA $(p, d, q)$ (Auto Regressive Integrated Moving Average) model [21, 22] are adopted to forecast the instantaneous parameters of OD flows, the prediction results as an estimation of normal pattern are subtracted by actual data so as to divide normal behavior, and the residual that represents the anomalous space is needed for the next correlation analysis.

Due to the strong correlation of two injected anomalies in time domain, as shown in Figure 4, we extract the anomalous space of instantaneous amplitude through our method, the result is shown in Figures Figure 7(a) and Figure 7(b), the similar changing tendency features of anomalies in instantaneous amplitude are captured accurately. This similar characteristic will contribute to strong correlation of anomalies, it will be introduced in the Section 6 .

\section{Network-Wide Correlation Analysis}

6.1. Network-Wide Correlation Analysis for Anomalous Space of OD Flows. The correlation of anomalous space from two different OD flows in time or frequency domain can be measured by correlation coefficient in statistical, which is defined as follows.

Let $X$ and $Y$ stand for two random variables, the covariance of $X$ and $Y$ is $\operatorname{Cov}(X, Y)=E\{[X-E(X)][Y-$ $E(Y)]\}$, where $D(X)$ and $D(Y)$ are the variance of $X$ and $Y$, respectively. The correlation coefficient of $X$ and $Y$ is computed by

$$
\rho_{x y}=\frac{\operatorname{Cov}(X, Y)}{\sqrt{D(X) D(Y)}} .
$$

The correlation coefficient is a measure of the linear relationship between two variables. The absolute value of $\rho_{x y}$ varies between 0 and 1 , with 1 indicating a perfect linear relationship, and $\rho_{x y}=0$ indicating no relationship.

Due to the path and delay in the network, the distributed anomalous flows may not rise in the same time, thereby it is not wise to consider the correlation of two anomalous space only in the same period. Two sliding windows are introduced to calculate the correlation coefficient between two neighborhood periods.

As shown in Figure 8, $O_{i}$ and $O_{j}$ are the anomalous spaces extracted from two different OD flows. Window $w 1$ starts at time $t$, intercepting the data of $O_{i}$ with length of $w 1$, as one of the vector. For the other anomalous space $O_{j}$, the window with start point varies between $(t-w 2, t+w 2)$, intercept the same length of data to be another vector. Every time the start point of window on $O_{j}$ moves, a correlation coefficient can 
be computed, the biggest one is output as coefficient of $\mathrm{O}_{i}$ and $O_{j}$ at time $t$ :

$$
\operatorname{coff}(i, j, t)=\operatorname{maxcorrcoef}\left[T_{i}(t), T_{j}\left(t_{j}\right)\right],
$$

where $t_{j}$ is the bound of start point of intercepted vector on $O_{j}$. Define the global correlation coefficient of the network at time $t$ as the mean of coefficients of all two OD flows:

$$
\text { Globalcoff }(t)=\frac{1}{m} \sum_{i} \sum_{j} \operatorname{coff}(i, j, t),
$$

where $m$ is the total number of $\operatorname{coff}(i, j, t)$, when $i \neq j$.

To detect anomaly accurately, a threshold is needed to determine whether the global correlation coefficient is abnormal. Study on history network traffic shows that the correlation coefficient of the network follows normal distribution. Therefore, coefficient's distribution in a period of historical time can be selected to set the baseline [23]. Assume in this period of time that the mean of coefficient is $m$, variance is $\delta$, standard variance is $\delta^{2}$, and threshold parameter is $\alpha$. The threshold $d$ is determined by

$$
d=m+\alpha * \delta .
$$

In $(8)$, set $\alpha=2.4$, confidence interval $m \pm 2.4 \delta$, confidence level of detect percent is $99.6 \%$, variance is $0.4 \%$. Compare the global correlation coefficient Globalcoff $(t)$ to the threshold, with

$$
\text { Globalcoff }(t) \geq d
$$

indicating anomaly at time $t$.

The global correlation coefficient of two anomalous spaces in instantaneous amplitude of no.26 and 50 OD flows in the Section 5 (see Figure 7) is shown in Figure 9. There is a pronounce spike about 1000 sample point, a sudden increase and close to 1 , and accurately capture the strong correlation between the anomalous space in instantaneous amplitude.

6.2. Error Analysis for Anomalous Space Extraction to Correlation Computation. Since the purpose of anomalous space extraction by prediction is to get the trend of traffic and extract the violation part from them, then examine whether there is correlation across multiviolation parts, rather than to get the precise predictions, thereby the accuracy of prediction algorithm is not the primary consideration in our method, but the simplicity and rapidness for the real-time detection. Since there are always deviation between the real and predictions, we now consider the influence of prediction error to the computation of correlation coefficient.

The anomalous space of every OD flows $R T_{t}$ consists of two components: the prediction error $e_{t}$ and the anomalous part $A_{t}$, namely, $R T_{t}=e_{t}+A_{t}$, when there exists no anomaly, $A_{t}=0$. For the anomalous space of two OD flows, $R T 1_{t}$, $R T 2_{t}$, the instantaneous parameters are forecasted independently, so the prediction errorse $1_{t}$ and $e 2_{t}$ are independent of each other. The prediction error and anomalous part which come from different OD flows, for instance, $e 1_{t}$ and $A 2_{t}$ or $e 2_{t}$ and $A 1_{t}$, are independent of each other. Therefore, it

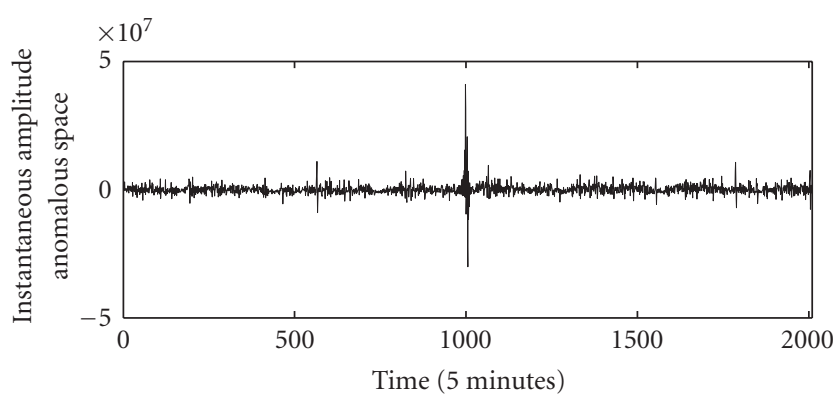

(a) Anomalous space of 26 OD flows

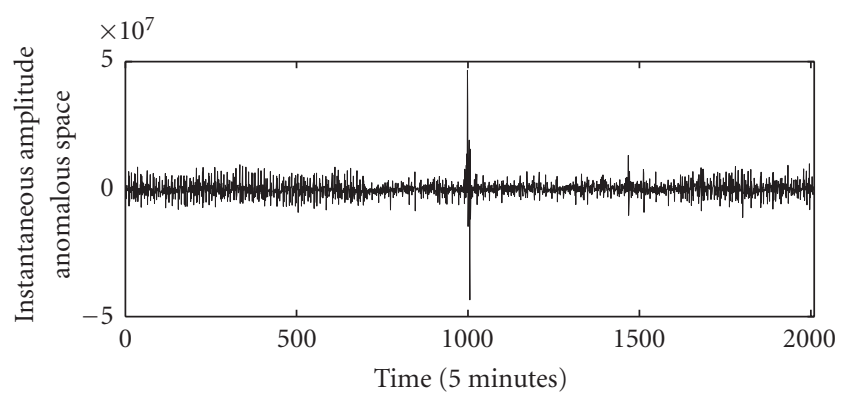

(b) Anomalous space of 50 OD flow

FIgURe 7: Anomalous space from instantaneous amplitude.

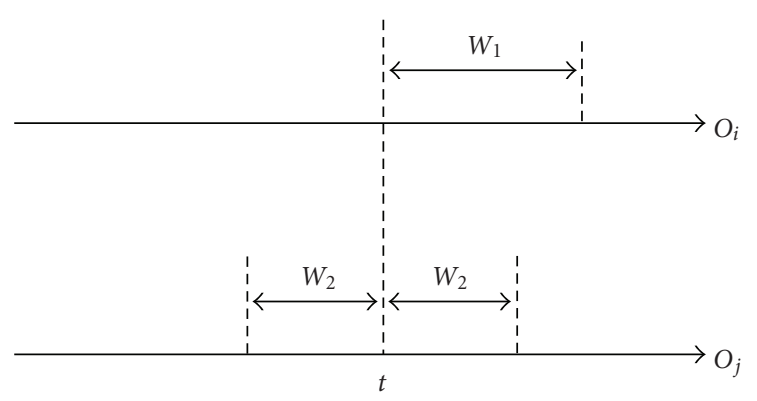

FIgURE 8: The correlation coefficient computation of two anomalous space.

can be proved that $\operatorname{Cov}\left(R T 1_{t}, R T 2_{t}\right)=\operatorname{Cov}\left(e 1_{t}+A 1_{t}, e 2_{t}+\right.$ $\left.A 2_{t}\right)=\operatorname{Cov}\left(A 1_{t}, A 2_{t}\right)$. Assuming that there are no prediction errorse $1_{t}=0, e 2_{t}=0$, the correlation coefficient of two OD flows can be rewritten as:

$$
\rho_{(R T 1)(R T 2)}=\rho_{(A 1)(A 2)}=\frac{\operatorname{Cov}(A 1, A 2)}{\sqrt{D(A 1) D(A 2)}}
$$

while prediction error does exist, the correlation coefficient is computed as

$$
\rho_{(R T 1)(R T 2)}=\frac{\operatorname{Cov}(R T 1, T 2)}{\sqrt{D(R T 1) D(R T 2)}}=\frac{\operatorname{Cov}(A 1, A 2)}{\sqrt{D(A 1) D(A 2)+\Delta}},
$$

where $\Delta=D(e 1) D(e 2)+D(e 1) D(A 2)+D(e 2) D(A 1)$ is nonnegative. To summarize, the more prediction errors, the smaller correlation coefficient will be. However in our method, we compute the correlation of every two anomalous 


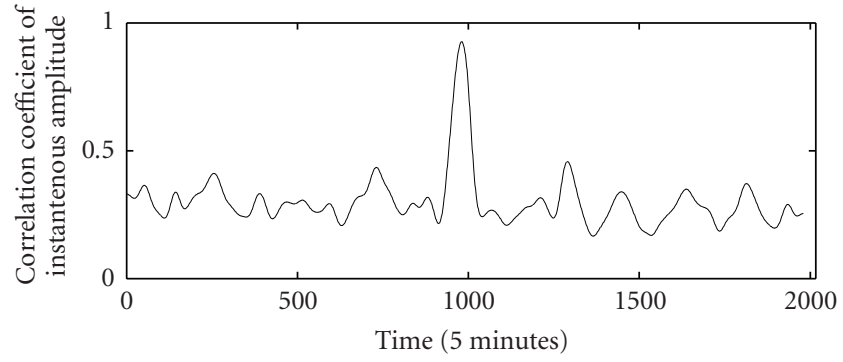

FIGURE 9: Global correlation coefficient of instantaneous amplitude in no.26, 50 OD flows.

space in the network, and all the coefficients are reduced compared with no error, it can be viewed as the overall effect. To eliminate the impact of the error, a statistical threshold of correlation coefficient in case of normal can be selected with the aim of comparison.

\section{Simulation and Analysis}

7.1. Simulation Data. The data for simulation in this paper is collected from American education backbone net Abilene by Yin Zhang [24], which has 11 Points of Presence (PoP) and 30 links in total. According to sample interval 1\%, we can get end-to-end data at each node and construct a time point every five minutes. Therefore, there are 2016 time points in each week. Totally 24 weeks' data are sampled from 2004-0301 to 2004-09-10.

7.2. Detection of Injected DDoS Attacks. To validate the power of our method, we apply our method to detect one of the distributed network anomaly-DDoS attack. In the simulation, we inject nonperiodic and periodic DDoS attacks. The attacks were added according to the following principles: the injected attacks are proportional to the mean of OD flows in which they are inserted; the attacks are unnoticeable compared to the volume of normal traffic; and they are not injected at the same time.

7.2.1. Nonperiodic DDoS Attack. According to the distributed characteristic of DDoS attacks, we choose node 4 as the node which is connected with victim's ISP, the OD flows $76,88,100,112,124,136$ are destined to this node. We randomly select three time points respectively near 400th, 900th, 1600th of above OD flows at the sixth week, as the start of anomalies being injected. At each beginning point, we insert three different attacks in turn which are noise, increasing rate attack, constant rate attack. Every attack lasts 100 sample points. The traffic before and after inserted attacks of 112 OD flows in the sixth week are depicted in Figure 10. The time intervals when attacks were injected have been marked by vertical dash lines.

The detection result of instantaneous amplitude is shown in Figure 11(a), in which the dotted line represents the correlation coefficient directly resulting from of the instantaneous amplitude of traffic, which means the correlation of normal

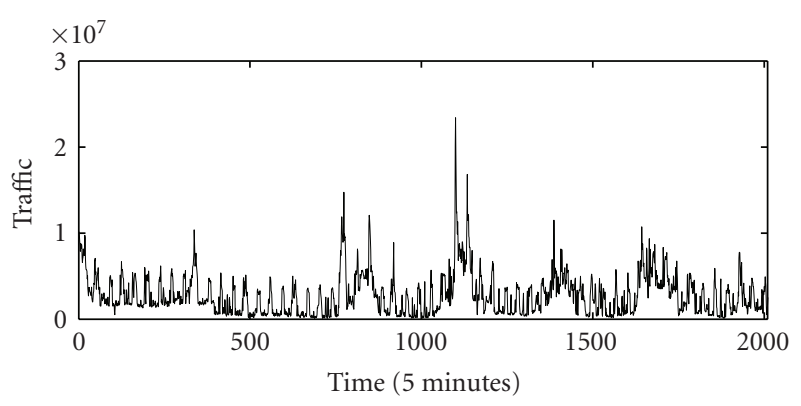

(a) Before injected attack

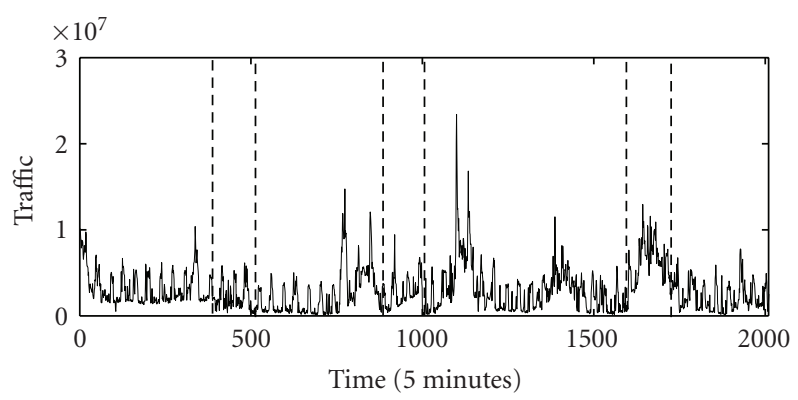

(b) After injected attack

FIGURE 10: 112 OD flow (6th week) before and after injected nonperiodic DDoS attacks.

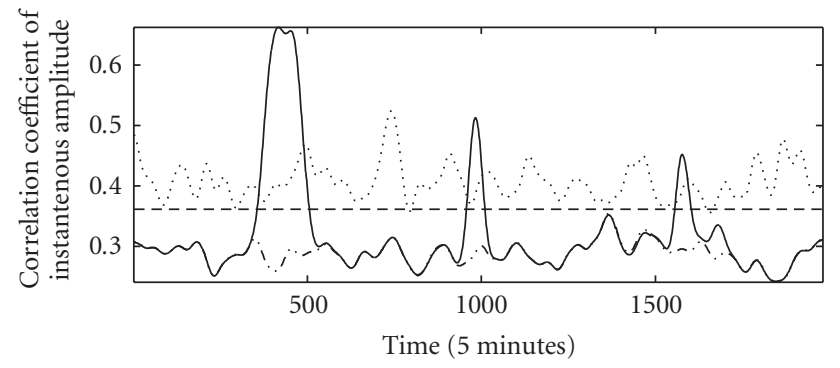

(a) The correlation coefficient of instantaneous amplitude

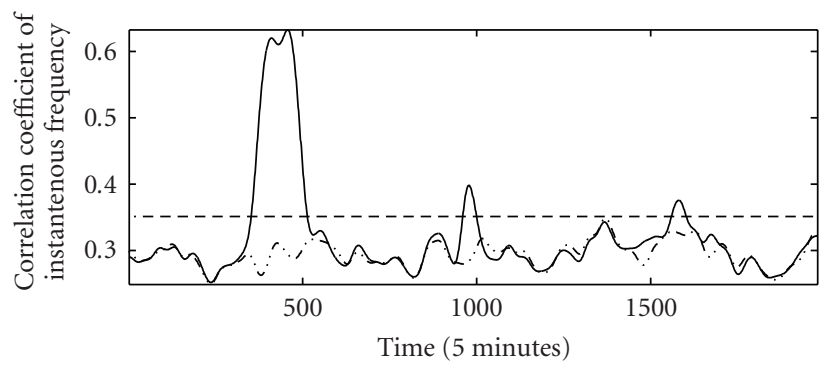

(b) The correlation coefficient of instantaneous frequency

FIGURE 11: The detection results of nonperiodic DDoS attacks.

traffic are not been cleared; the dash-dot line is the correlation coefficient of anomalous space without inserted attacks. Two observations are available from the dash-dot line (comparing with the dotted line): (1) it changes more moderate than coefficient resulting directly from the instantaneous amplitude 


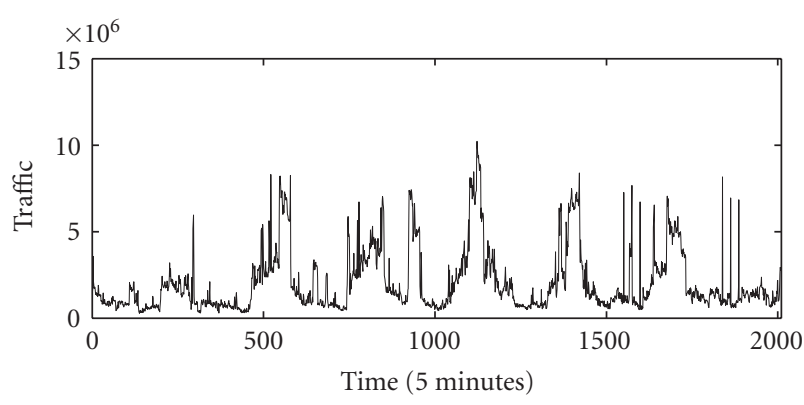

(a) Before injected attack

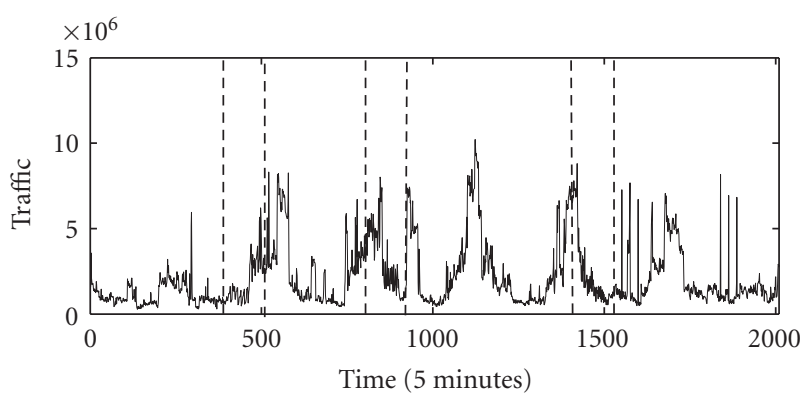

(b) After injected attack

Figure 12: 124 OD flow (17th week) before and after injected periodic DDoS attacks.

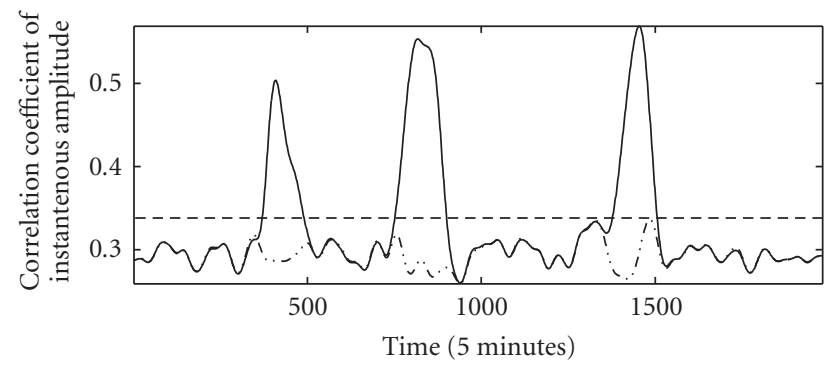

(a) The correlation coefficient of instantaneous amplitude

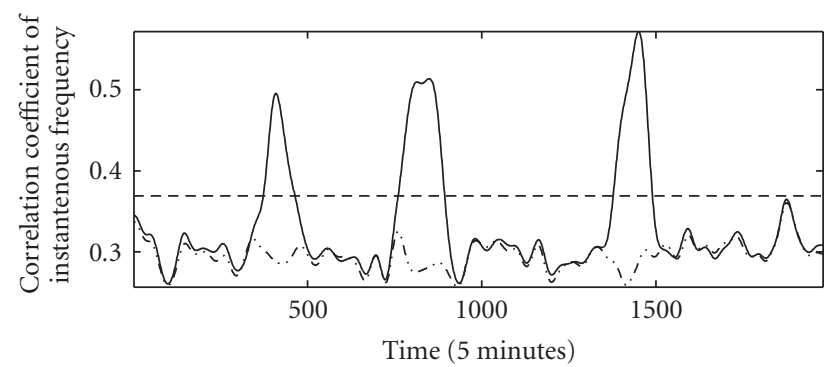

(b) The correlation coefficient of instantaneous frequency

FIGURE 13: The detection results of nonperiodic DDoS attacks.

of traffic (dotted line), because correlation parts in normal traffic is erased by subtracting predictions from actual data, accordingly the residual standing for random factor has less correlation than normal traffic. (2) a offset phenomenon of dash-dot line towards the bottom, it consists with our reasoning before that the prediction error causes a overall effect to the computation of coefficient.

After the attacks were injected, the correlation coefficient (solid line) changes drastically and surpass the threshold (horizontal dash line) (set $\alpha=3$ ) during each period of attacks were injected. The work of [19] has showed there are difference between the instantaneous frequency of anomalous traffic and that of normal, therefore it can be used as a feature to detect some part of network attacks. In our work, we discover a few anomalies tend to cause relatively smaller impact to instantaneous frequency of background traffic than influence on instantaneous amplitude, or vice versa. As shown in Figure 11(b), the detection results of instantaneous frequency to increasing rate and constant rate attack, correlation coefficient corresponding to the attack were injected exceeds the threshold and are smaller than that of instantaneous amplitude (Figure 11(a)). This is due to attackers can easily change the pattern of attack during attack is launched, anomalous traffic may cause different impact to instantaneous frequency and instantaneous amplitude of background traffic. For this reason, a better detection performance could be achieved by combining the analysis of instantaneous frequency and instantaneous amplitude of traffic.

7.2.2. Periodic DDoS Attack. The periodic DDoS attacks are injected in 17th week traffic data, target node 4 . Nearby the time points 400th, 800th, 1400 th of $76,88,100,112,124,136$ OD flows respectively been inserted: periodic increasing rate attack, middle frequency attack, high frequency attack. Each lasts 100 time points. In Figure 12 is the traffic of 124 OD flows before and after added attacks.

As the periodic feature of attacks in time domain, there are same intrinsic property in frequency (such as the same frequency components) between the attacks, which leads to well performance in instantaneous frequency, as depicted in Figure 13(b), the correlation coefficient (solid line) drastically exceeds the threshold (horizontal dash line). Furthermore, periodic attacks also can be revealed by instantaneous amplitude due to the periodicity in time domain, as shown in Figure 13(a). As a result, simultaneously analyzing the correlation of time domain and frequency domain can increase the possibility of detecting attacks.

7.3. Detection of Real Distributed Anomaly. As we employed our method on the original Abilene data, without injected attack, some detection results attracted our attention, the coefficient of both instantaneous amplitude and frequency in the third week nearby 1420th sample point, surpass the threshold (Figures 14(a), 14(b)). Studying the traffic of this week, we found that $77,89,101,113,125,137$ OD flows which have the same destination node 5 , appear to suddenly fall or rise at the 1420th sample point, as marked between vertical dash lines in Figure 15. The traffic pattern is different from other weeks. With the experience of network operators, there might be a anomalous event (e.g., outage, egress shift, failure) happening in a certain node or link, where 77, 89, 113,125 OD flows both passed by and resulted in these 


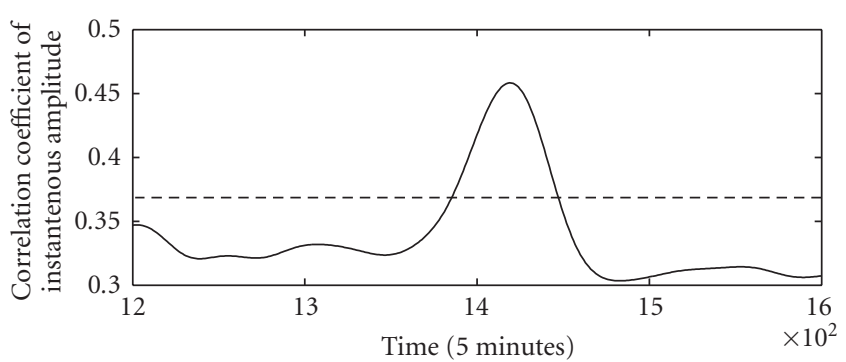

(a) The coefficient of instantaneous amplitude

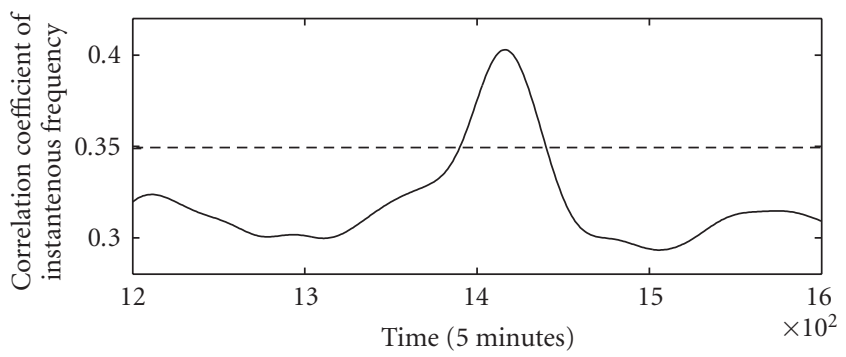

(b) The coefficient of instantaneous frequency

Figure 14: The detection results of 3rd week.

traffic falling down; at the same time, the OD flows that directed to node 5 had to bypassed this node or link, and arrived at their destination through other path, consequently the traffic of 101, 137 OD flows rose up. These OD flows were changed abruptly by the same reason, and formed distributed traffic anomalies. The results of this experiment show that our method still works well in detecting real distributed anomalies of network traffic.

7.4. Comparison with PCA-Based Method. PCA-based detection method decomposes network-wide traffic into normal space and anomalous space with the help of PCA, the latter is also referred to as residual part. The essence of PCA is a dimension reduction method in the mean-square sense. More specifically, given the dimension of data set is $m$, the objective of PCA is to find a few orthogonal principle axes $w_{1}, w_{2}, \ldots, w_{n}(n<m)$, so that the projection on these directions, namely principle component, is as faithful as possible to capture the variance of original data, the first principle axis points in the direction of maximum variance in original data, other principle axes which in turn point in the directions of maximum variance remaining in data. The basis of PCA-based method which divides anomalous space is to examine the projection on every principal axis in order, if projection containing $3 \delta$ deviation from the mean is found, the projection on this principal axis and all subsequent axes are considered as residual part, once the squared magnitude of residual part surpasses a threshold deriving from Q-statistic, an alarm is triggered.

In Section 5, we have showed that PCA-based method tends to assign the anomalies with strong correlation into normal space. Now we perform PCA method described in [1] to detect the same injected nonperiod and period DDoS attacks. The residual parts divided by PCA are respectively
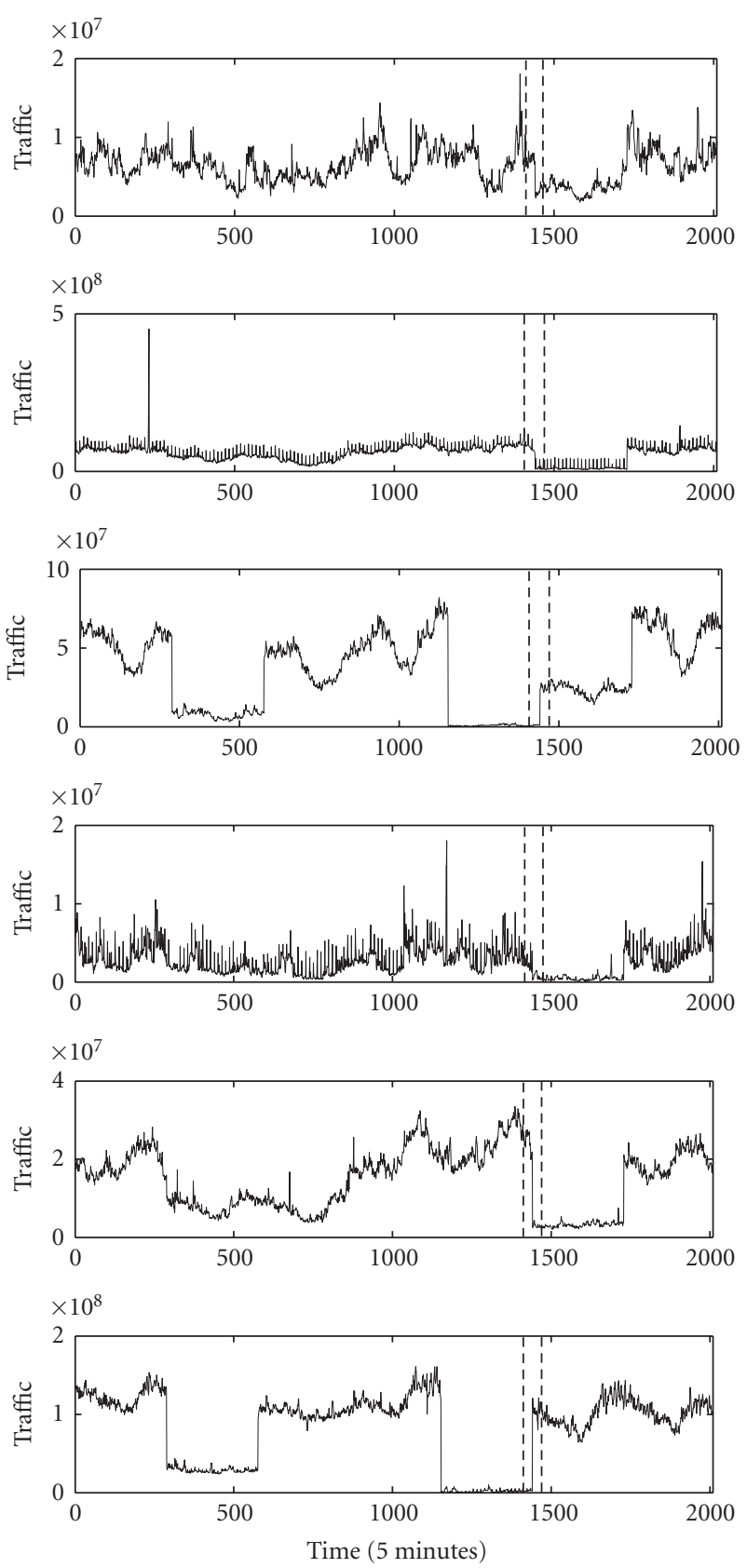

Figure 15: 77, 89, 101, 113, 125, 137 OD flows of 3rd week.

presented in Figures 16(a) and 16(b), in which the solid lines represent the residual parts after injected attacks while the dash-dot lines are that of original data. A zoom is shown as an insideplot in Figure 16(b).

During the period when attacks were inserted, as marked with vertical dotted lines, the residual parts of before and after attacks were injected almost superpose upon each other, they show that PCA-based method did not assign anomalies into residual part. As the residual parts divided from which attacks were inserted (corresponding to solid lines in both figures), are far less than thresholds which represented by horizontal dash lines on the top of the figures, alarm could not be triggered. 


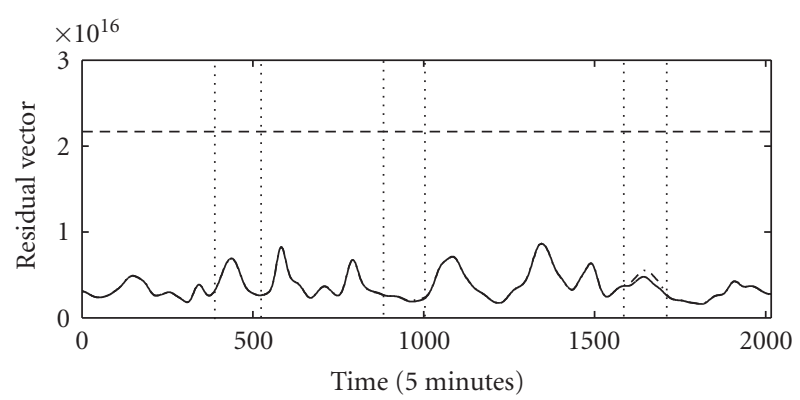

(a) Non-period DDos attack (before injected attacks (dash-dot line), after injected attack (solid line))

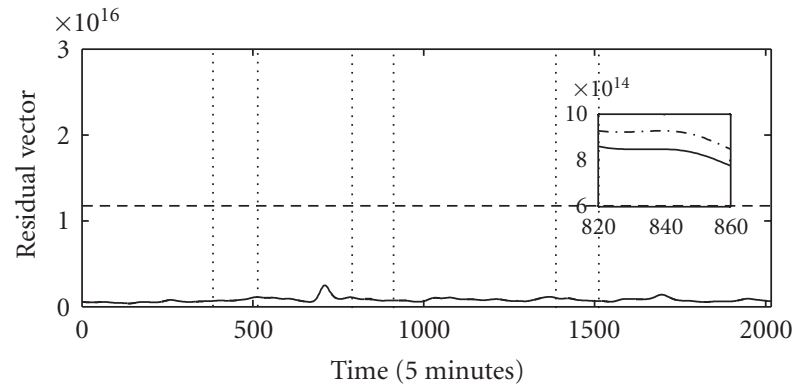

(b) Period DDos attack (before injected attacks (dash-dot line), after injected attack (solid line))

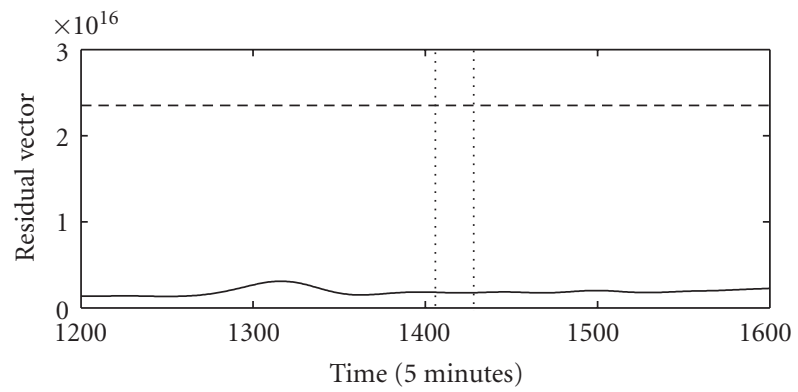

(c) Analysis of 3rd week

FIgURE 16: The detection results based on PCA.

The residual part of real distributed anomalies in 3rd week separated by PCA is shown in Figure 16(c). As anomalies occurring (marked with vertical dotted lines), the residual vector does not change obviously and retains lower than threshold (corresponding to horizontal dash line). As a result, PCA-based method cannot reveal the anomalies which appear in multi OD flows simultaneously.

\section{Conclusion}

Distributed traffic anomaly is small in single link and hard to detect while total volume of anomalies in multiple links is great and anomalous traffic signals of each link are similar in time or frequency domain. Aiming at its similar features between different links, we propose a method to detect distributed network traffic anomaly with network-wide correlation analysis of instantaneous parameters. We experimented with different anomalous modes: non-periodic DDoS attacks, periodic DDoS attacks and real distributed anomaly. Our simulation results show that: (1) Instantaneous amplitude and instantaneous frequency of traffic signal can reveal anomalies by its characteristics of time and frequency domain; (2) Our anomalous space extraction method based on traffic prediction can overcome the limitations of PCA-based method in failing to detect the anomalies with strong correlations; (3) the network-wide correlation analysis of amplitude and frequency can detect distributed network traffic anomaly while anomaly in single link is very small.

\section{Acknowledgments}

The authors would like to thank Yin Zhang for providing us with Abilene traffic data. The work described in this paper was supported by NSFC (Project no. 60872033), Program for New Century Excellent Talents in University(NCET-070148), and The National Key Basic Research Program of China "973Project" (2007CB307104 of 2007CB307100).

\section{References}

[1] A. Lakhina, M. Crovella, and C. Diot, "Characterization of network-wide anomalies in traffic flows," in Proceedings of the ACM SIGCOMM Internet Measurement Conference (IMC '04), pp. 201-206, Taormina, Italy, October 2004.

[2] A. Lakhina, M. Crovella, and C. Diot, "Diagnosing networkwide traffic anomalies," in Proceedings of the ACM SIGCOMM Conference on Applications, Technologies, Architectures, and Protocols for Computer Communications, pp. 219-230, Portland, Ore, USA, August-September 2004.

[3] K. Park and H. Lee, "On the effectiveness of route-based packet filtering for distributed DoS attack prevention in power-law Internets," in Proceedings of the ACM SIGCOMM Conference on Applications, Technologies, Architectures, and Protocols for Computers Communications, pp. 15-26, San Diego, Calif, USA, August 2001.

[4] R. K. C. Chang, "Defending against flooding-based distributed denial-of-service attacks: a tutorial," IEEE Communications Magazine, vol. 40, no. 10, pp. 42-51, 2002.

[5] K. K. Wan and R. Chang, "Engineering of a global defence infrastructure for DDoS attacks," in Proceedings of the 10th IEEE International Conference on Networks (ICON '02), pp. 419-427, Singapore, August 2002.

[6] D. Sterne, K. Djahandari, B. Wilson, et al., "Autonomic response to distributed denial-of-service attacks," in Proceedings of the 4th International Symposium on Recent Advances in Intrusion Detection (RAID '01), pp. 134-149, Davis, Calif, USA, October 2001.

[7] D. Schnackenberg, K. Djahandari, and D. Sterne, "Infrastructure for intrusion detection and response," in Proceedings of the DARPA Information Survivability Conference and Exposition (DISCEX '00), vol. 2, pp. 3-11, Hilton Head, SC, USA, January 2000.

[8] Q. Zhang and R. Janakiraman, "Indra: a distributed approach to network intrusion detection and prevention," Tech. Rep. WUCS-01-30, Washington University, Saint Louis, Mo, USA, 2001.

[9] G. Koutepas, F. Stamatelopoulos, and B. Maglaris, "Distributed management architecture for cooperative detection and reaction to DDoS attacks," Journal of Network and Systems Management, vol. 12, no. 1, pp. 73-94, 2004. 
[10] J. Ioannidis and S. Bellovin, "Implementing pushback: routerbased defense against DDoS attacks," in Proceedings of the Network and Distributed System Security Symposium (NDSS '02), San Diego, Calif, USA, February 2002.

[11] Y. Chen and K. Hwang, "Collaborative detection and filtering of shrew DDoS attacks using spectral analysis," Journal of Parallel and Distributed Computing, vol. 66, no. 9, pp. 11371151, 2006.

[12] A. Lakhina, M. Crovella, and C. Diot, "Mining anomalies using traffic feature distributions," in Proceedings of the ACM SIGCOMM Conference on Applications, Technologies, Architectures, and Protocols for Computer Communications, pp. 217-228, Philadelphia, Pa, USA, August 2005.

[13] A. Lakhina, M. Crovella, and C. Diot, "Detecting distributed attacks using network-wide flow traffic," in Proceedings of the FloCon Analysis Workshop (FloCon '05), Pittsburgh, Pa, USA, September 2005.

[14] X. Li, F. Bian, M. Crovella, et al., "Detection and identification of network anomalies using sketch subspaces," in Proceedings of the 6th ACM SIGCOMM on Internet Measurement Conference (IMC '06), pp. 147-152, Rio de Janeriro, Brazil, October 2006.

[15] J. Yuan and K. Mills, "Monitoring the macroscopic effect of DDoS flooding attacks," IEEE Transactions on Dependable and Secure Computing, vol. 2, no. 4, pp. 324-335, 2005.

[16] Y. Huang, N. Feamster, A. Lakhina, and J. Xu, "Diagnosing network disruptions with network-wide analysis," in Proceedings of the International Conference on Measurement and Modeling of Computer Systems (SIGMETRICS '07), pp. 61-72, San Diego, Calif, USA, June 2007.

[17] C.-M. Cheng, H. T. Kung, and K.-S. Tan, "Use of spectral analysis in defense against DoS attacks," in Proceedings of IEEE Global Telecommunications Conference (GLOBECOM '02), vol. 3, pp. 2143-2148, Taipei, Taiwan, November 2002.

[18] A. Hussain, J. Heidemann, and C. Papadopoulos, "A framework for classifying denial of service attacks," in Proceedings of the ACM SIGCOMM Conference on Computer Communications, pp. 99-110, Karlsruhe, Germany, August 2003.

[19] W. Wang, K. Pei, X. Jin, et al., "Using Hilbert-Huang transform to characterize intrusions in computer networks," in Proceedings of the 3rd International Conference on Natural Computation (ICNC '07), vol. 5, pp. 749-753, Haikou, China, August 2007.

[20] N. E. Huang, Z. Shen, S. R. Long, et al., "The empirical mode decomposition and the Hilbert spectrum for nonlinear and non-stationary time series analysis," Proceedings of the Royal Society of London, vol. 454, no. 1971, pp. 903-995, 1998.

[21] K. Papagiannaki, N. Taft, Z.-L. Zhang, and C. Diot, "Longterm forecasting of Internet backbone traffic: observations and initial models," in Proceedings of the 22nd Annual Joint Conference on IEEE Computer and Communications Societies (INFOCOM '03), vol. 2, pp. 1178-1188, San Francisco, Calif, USA, March 2003.

[22] G. E. Box and G. M. Jenkins, Time Series Analysis: Forecasting and Control, Holden Day, San Francisco, Calif, USA, 1976.

[23] S. S. Kim, A. L. N. Reddy, and M. Vannucci, "Detecting traffic anomalies through aggregate analysis of packet header data," in Proceedings of the 3rd International IFIP-TC6 Networking Conference on Networking Technologies, Services, and Protocols; Performance of Computer and Communication Networks; Mobile and Wireless Communications (Networking '04), pp. 1047-1059, Athens, Greece, May 2004.

[24] http://www.cs.utexas.edu/ yzhang/research/AbileneTM/. 